\title{
Evaluation of Energy Efficient Reactive Routing Protocols in QoS Enabled Routing for MANETS
}

\author{
N.Sumathi \\ S.N.R.Sons College, Coimbatore
}

\author{
Dr. Antony Selvadoss Thanamani \\ Head, Dept of Computer Science, \\ N.G.M. College, Pollachi
}

\begin{abstract}
A mobile node has a finite and decreasing energy. When finding routes, energy aware routing increases the network lifetime. Energy saving mechanism is required for the efficient operation of the battery powered network. In general, all the neighboring nodes overhear transmission when a node is transmitting a packet. Overhearing is caused by receiving information that is not addressed to it. Energy consumed by overhearing nodes is same as that of receiving nodes. The main aim of this work is to develop an energy conserving mechanism to reduce energy expenditure due to overhearing. To minimize energy, number of overhearing nodes is randomly selected based on probability. This probability based overhearing is integrated with AODV routing protocol. This proposed approach is implemented in NS2 simulator. Simulation results are presented to demonstrate the performance metrics such as throughput, packet delivery ratio, end-end delay and energy consumption of the network.
\end{abstract}

\section{General Terms}

Mobile Ad hoc Networks, QoS Routing.

\section{Keywords}

QoS Routing, Energy, AODV, Overhearing, DSR.

\section{INTRODUCTION}

Mobile ad hoc networks (MANETs) can be deployed wherever there is no or little communication infrastructure is available. They consist of battery operated mobile nodes that communicate through wireless medium. These nodes need to be energy conserved to maximize the battery life. A node communicates directly with nodes in the transmission range and indirectly with other nodes in communication range. A MANET is characterized by bandwidth and energy-constrained mobile nodes with variablecapacity links. It has dynamic, unpredictable topology. A mobile node has a finite, decreasing energy. Energy management is done by MAC layer using local information while the network layer can take decisions based on topology or traffic characteristics. Energy consumed by the sleeping state node is significantly less than the transmit/receive/idle state node. To minimize energy consumption, path which consumes less power is chosen.

The infrastructure less environment of ad hoc networks poses constraints on routing protocols. Ad hoc routing protocols can be broadly classified as proactive (table driven) reactive (on demand) and hybrid [1]. Proactive approach costs high to transmit the topology information. Routes are calculated in advance, independent of current traffic. The reactive approach, on the other hand, reduces routing overhead so that bandwidth is saved.
They execute the routing process and exchange routing information only when there is a demand for a node to transmit data to some destination. Brown et al. [2] show that the proactive schemes are more expensive in terms of energy consumption as compared to the reactive schemes because of the large routing overhead in the former. Therefore reactive routing protocols are given importance in this work.

Two mostly used reactive protocols are Ad hoc On-demand Distance Vector (AODV) [1] and Dynamic Source Routing (DSR) $[3,4,5]$. Both are uniform protocols, in which all nodes participate equally in the routing process: there are no distinguished nodes and no hierarchical structure is imposed on the network. These protocols differ primarily in the state information that they maintain. DSR is a source routing protocol. Only the source node knows the whole route to the destination. Each data packet stores the whole route information in the header which costs high overhead and wastes bandwidth. There is a chance of using stale routes which cause loss of packets because there is no timer information. AODV is a destination-oriented protocol based on the distributed Bellman-Ford algorithm. Each node stores the information of the next hop and destination in its routing table. Timer is used to maintain the freshness of a route. It provides only one route reply. Also one route entry per destination is stored in the routing table. It maintains distancevector routing information and uses a destination managed sequence number to avoid routing loops.

This paper is structured as follows. Section 2 describes related work on energy conserving techniques. Section 3 presents probability based overhearing concept and its integration with AODV routing protocol. Section 4 show simulation results and section 5 concludes the paper.

\section{RELATED WORK}

Wireless ad hoc networks use a wide range of energy conserving techniques. In AFECA (Adaptive Fidelity Energy Conservation Algorithm), nodes sleep based on the size of their neighborhoods [6]. When number of neighbors is more, then node enters into sleep state without disconnecting the network. Reference [7] provides different sleep patterns for the mobile nodes based on their residual energy and quality of service. But a special hardware, called Remote Activated Switch (RAS), is required to wake up the sleeping nodes. Technique to combine power management and power control for wireless cards is presented in [8]. GAF (Geographic Adaptive Fidelity) discussed in [9] uses GPS to form a grid such that adjacent boxes are guaranteed to be within the communication range of each other. Then, selected nodes in each box remain on to keep the network connected. 
In power conserving algorithm [4], a node enters into the doze state if it overhears RTS/CTS for data transmission. However, this approach incurs more transition costs associated with a doze-toactive transition. In PSM [10] (Power Saving Mode) of 802.11, when a node transmits or receives an ATIM (Ad hoc Traffic Indication Map) frame during an ATIM window, it must be in active mode during the entire beacon interval that results in a much higher energy consumption. In DPSM [10] (Dynamic Power Saving Mechanism) scheme, the ATIM window size is adjusted dynamically based on current network conditions. A NPSM [11] (New Power Saving Mechanism) introduces some parameters indicating amount of data in each station. Powersaving mechanism is hard to implement in partial connected like mobile multi-hop network. In [12], off-the-shelf hardware is used to wake up wireless devices in a centralized environment. In [13], energy is saved by integrating routing and MAC layer functionality. However, the timers do not adjust to the traffic rate, so if traffic is not frequent enough to refresh the timers, the benefits of the protocol are lost. Even though GEAR [14] (Global Energy Aware Routing) can save energy and maximize the system lifetime; it has two major disadvantages (difficulty in utilizing route cache and the blocking property) that can be avoided with Local Energy-Aware Routing (LEAR) protocol. In ODPM (On Demand Power Management) [13], soft state timers are set or refreshed on-demand, based on control messages and data transmission. Nodes that are not involved in data transmission may enter into sleep state to save energy. It shows smallest power latency. This is because nodes transmit data packets immediately without waiting for the next beacon interval.

The protocol discussed in [15] extends doze time and reduces contention, retransmission and improves channel utilization. It also provides quality of service support. In [16], number of AM nodes is reduced based on backbone probability. TITAN (TrafficInformed Topology-Adaptive Network) [17] improves ODPM in which PS nodes sleep for longer duration and saves energy. Rcast [18] implements randomized overhearing but not randomized rebroadcast. It uses pseudo-ACK packet to inform that it can forward the packet during the subsequent beacon interval. Dorsey and Siewiorek [19] discussed a fast wakeup mechanism so that latency for route discovery is reduced. Randomcast [20] uses no/unconditional/ randomized overhearing depending on the packet type and introduces randomized rebroadcast to improve performance. It reduces energy consumption and improves energy balance. This algorithm is integrated into DSR routing protocol. In DSR, overhearing leads to bad situation because stale route concept is applied to all unconditional overheard nodes and wastes energy resource while transmitting, receiving, rebroadcast and unconditional overhearing. DSR broadcasts control packets which waste channel capacity because it generates redundant rebroadcasts. Hence AODV routing protocol is proposed.

\section{ENERGY EFFICIENT AODV}

Overhearing improves routing efficiency but consumes energy. Overhearing is caused by the fact that when a unicast packet transaction is carried out in a node's immediate neighborhood, it does not have any mechanism for not to receive that packet. Energy expenditure during overhearing is same as that during reception. Goal of this work is to develop an energy conserving mechanism to reduce energy expenditure due to overhearing. Overhearing improves network performance since nodes collect more route information. Nodes in the range of source learn about path to destination. But overhearing increases traffic and consumes energy. The proposed algorithm controls the level of overhearing. It reduces energy consumption without affecting quality of route information.

Overhearing and redundant rebroadcast problems are controlled using ATIM window. RTS, CTS, ATIM, Beacon and ACK frames are allowed to transmit during the ATIM window. MSDU (MAC Service Data Unit) frames and other management frames needs to be transmitted out side the ATIM window. ATIM frame consists of frame control, destination address, source address, sequence control etc. Subtype field in frame control helps to set the overhearing level.

Subtype: 1001 for unconditional overhearing

\section{1 for probability based overhearing}

1110 for no overhearing

This algorithm enables the sender to select no overhearing, unconditional overhearing or probability based overhearing for its neighbors. It is specified in the ATIM frame's sub type field and made it available to its neighbors during ATIM window. Number of overhearing nodes is controlled by probability based overhearing method.

\subsection{Function of probability based overhearing}

Probability based overhearing method controls the level of overhearing and forwarding of broadcast messages. Sender is able to specify the level of overhearing. Sender may choose no or unconditional or probability overhearing which is specified in ATIM frame control. Node is awakened if unconditional overhearing or probability overhearing is set or it is a destination node. Each node maintains overhearing probability $\mathrm{Po}$ and rebroadcast probability $\mathrm{Pr}$.

$\mathrm{Po}=1 / \mathrm{n}$

$\operatorname{Pr}=\mathrm{cn} / \mathrm{N}^{2}$

Where $\mathrm{c}$ is a constant (4.0), $\mathrm{n}$ is no. of neighbors and $\mathrm{N}$ is average no. of neighbor's neighbors.

$\mathrm{Ec}=\sum(\mathrm{Ie}-\mathrm{Re}) /$ no. of pkts sent

where Ec is energy consumed, Ie is Initial energy and Re is residual energy.

If a node's subtype is 1101 , it generates a random number between 0 and 1 and compares it with Po. If it is greater than Po, node decides to overhear. If it is greater than Pr, node decides to rebroadcast. Po and $\mathrm{Pr}$ are decided based on number of neighbors. When the number of neighbors is more, redundancy is more. Figure 1 shows that $\mathrm{A}$ and $\mathrm{B}$ are overhearing nodes. They overhear the transmissions between the nodes $\mathrm{S}$ and D. Other neighbor nodes are in sleep state.

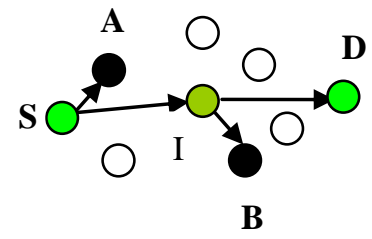

Figure 1. Probability based overhearing 


\subsubsection{Steps to compare overhearing level and set subtype}

When a node is ready to transmit a frame, check its overhearing level (OL) and set its sub type for broadcast and unicast transmission.

1. For a broadcast transmission

If $(\mathrm{OL}=$ unconditional $)$

Send ATIM.

Else if $(\mathrm{OL}=$ probability overhearing $)$

If (rand $(0,1) \leq \operatorname{Pr}$ ) send ATIM.

2. For a unicast transmission

If $(\mathrm{OL}=$ unconditional $)$

Subtype $=1001$.

Else if $(\mathrm{OL}=$ no overhearing $)$

Subtype $=1110$.

Else if $(\mathrm{OL}=$ probability overhearing $)$

Subtype $=1101$.

Send ATIM with subtype.

After receiving an ATIM frame, node decides to receive or overhear or to be in sleep mode in the following data transmission period based on destination address and subtype values.

1. If it is a destination node then

Receive the packet.

else check its subtype

a. If subtype $=1001 / /$ unconditional

Overhear transmission.

b. If subtype $=1101 / /$ probability overhearing If $(\operatorname{rand}(0,1) \leq \mathrm{Po}$

Node decides to overhear the transmission.

c. If subtype $=1110 / /$ No overhearing

Go to sleep state.

This probability based overhearing is integrated into a routing protocol called AODV to find the route from a given source to destination.

\subsection{Routing Protocol}

The main objective of AODV routing protocol is to find a route that satisfies QoS request. Each node in the network consists of two data structures such as routing table and neighbor list. Routing table at each node stores the list of reachable nodes and their bandwidth. It consists of destination_id, next_id and bandwidth. Neighbor list is used to store the information of all the neighboring nodes. The RREQ(Route REQuest) contains sourceaddr, source-sequence\#, dest-addr, dest-sequence\#,hop-cnt and an extension field bandwidth. The routing algorithm is able to detect the dynamic topology and generates path between nodes. It should also handle route failures. The routing is performed in three phases. They are:

- Route discovery phase: This phase finds all possible paths from source node to destination node.

- Route maintenance phase: This phase strengthens the path between the nodes.

- QoS Loss recovery: If any node along the source to destination fails or moves away from the network, alternate paths will be generated.

\section{SIMULATION RESULTS}

The proposed algorithm is implemented using NS2 simulator tool [21]. Performance analysis of energy efficient AODV is carried out by setting simulation time to 200 s with a grid size of $1000 \times 1000 \mathrm{~m}$. Random way point mobility model with CBR (Constant Bit Rate) traffic is used to simulate nodes movement. This algorithm is tested with 30 nodes. The parameters used to measure the performance are number of packets received, delay, energy consumption and packet delivery ratio. Table 1 shows simulation parameters. The results are compared with existing DSR protocol.

Table 1. Simulation Parameters

\begin{tabular}{|l|l|}
\hline Parameter & Value \\
\hline Transmission range & $250 \mathrm{~m}$ \\
\hline Carrier Sensing range & $550 \mathrm{~m}$ \\
\hline Packet Size & 512 bytes \\
\hline Channel Capacity & $2 \mathrm{Mbps}$ \\
\hline Grid Size & $1000 \times 1000 \mathrm{~m}$ \\
\hline No. of nodes & 30 \\
\hline Mobility Speed & $20 \mathrm{~s}$ \\
\hline Simulation Time & $200 \mathrm{~s}$ \\
\hline
\end{tabular}

Figure 2 shows the number of packets received by AODV and DSR method. Number of packets received in energy efficient AODV is more than DSR.

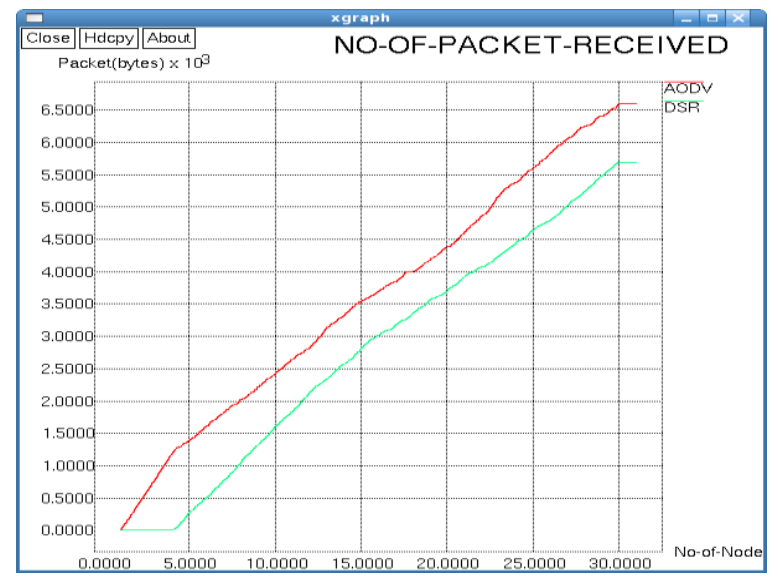

Figure 2. Packets Received

The packet delivery ratio (PDR) is calculated as the ratio of the data packets delivered to the destination to those transmitted by the CBR traffic. The ability to deliver a high percentage of packets to a destination increases the overall utility of the system. PDR is high because most of the nodes are participated in packet transmission as shown in figure 3. PDR is reduced due to the mobility of nodes and link failures. It is observed that proposed method maintains a significantly high PDR than the existing one. Because the most active path is selected, this has less probability to fail. 


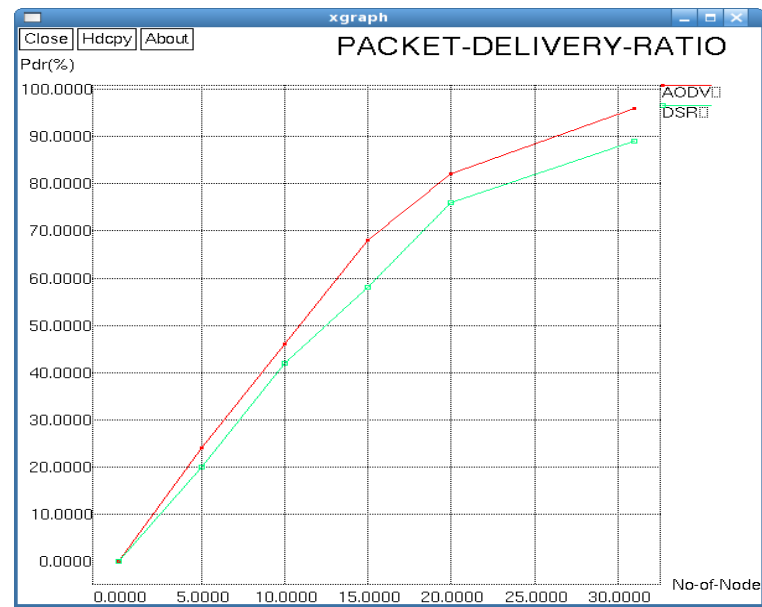

Figure 3. Packet Delivery Ratio

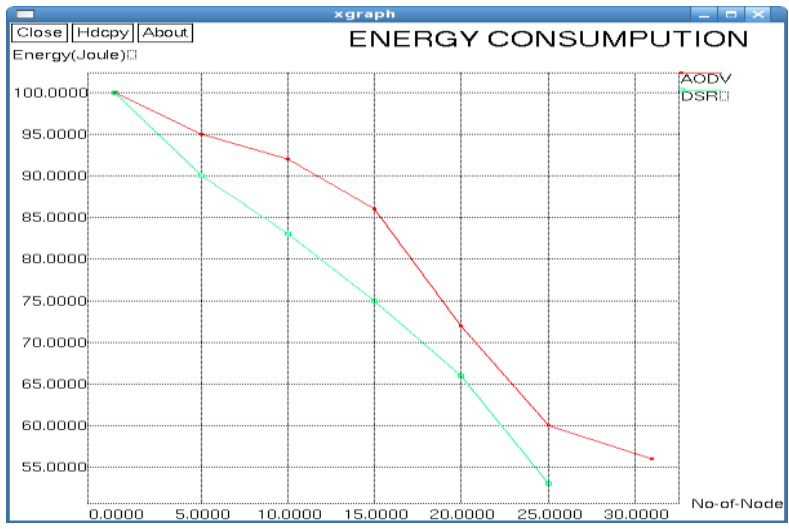

Figure 4. Energy Consumption

Probability based overhearing method outperforms other algorithms with respect to energy consumption. Energy efficient AODV shows less energy consumption than DSR as in Figure 4. Energy efficient scheme achieves better energy performance under high traffic condition. Performance gap is not dramatic under low traffic condition. DSR consumes more energy since nodes are awake during the entire period of simulation time. In AODV energy efficient scheme, nodes in the range of active communication overhear probabilistically. Effective energy management is obtained in proposed work. This is due to the variation of the transmit power between two nodes and also reduction in the number of overhearing nodes. This proposed approach with energy management still reduces the energy consumption.

Average end-end delay is calculated based on the average time required to transmit packets from the source to destination. This end-end delay includes delays caused by buffering during route discovery latency, queuing, retransmission delays, propagation and transfer times. Figure 5 shows that the delay caused by energy efficient AODV is less than DSR.

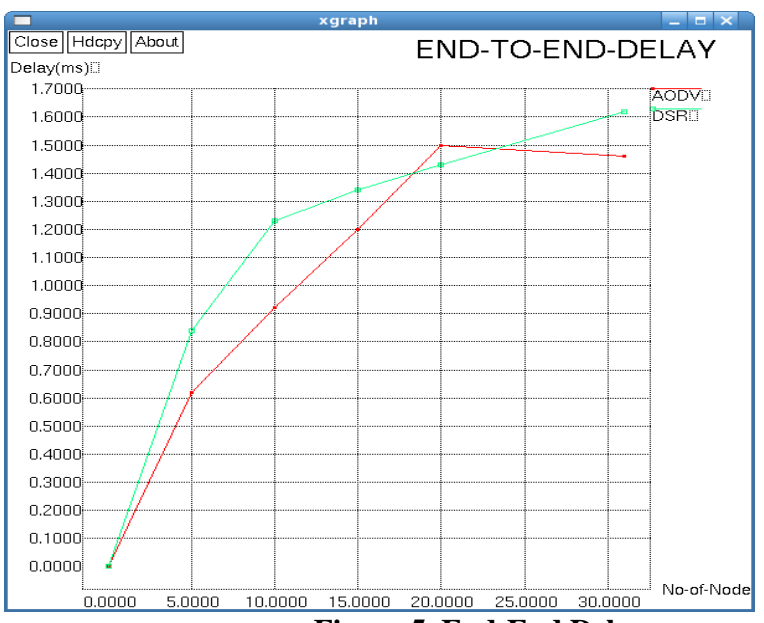

Figure 5. End-End Delay

\section{CONCLUSION}

Energy is saved by integrating MAC layer functionality with routing. Sender can specify the desired level of overhearing based on overhearing probability. Hence the energy consumed by overhearing nodes is reduced. Problem of unconditional or unnecessary forwarding of broadcast packets are considered. This probability based overhearing is incorporated into AODV to save energy. This paper compares the performance of energy efficient AODV with DSR in terms of throughput, PDR, delay etc. Hence the proposed method outperforms the existing one. It is implemented in NS2 simulator to verify the changes in parameters such as packets received, packet delivery ratio, end to end delay and energy consumption for different number of nodes.

\section{REFERENCES}

[1] C.E. Perkins and E.M. Royer, Ad-hoc on-demand distance vector routing, in: Proceedings of the 2nd IEEE Workshop on Mobile computing Systems and Applications, New Orleans, LA (February 1999) pp. 90-100.

[2] Brown, T.X, Doshi, S., Zhang, Q., "Optimal power aware routing in a wireless ad hoc network," IEEE LANMAN 2001 Workshop Proceedings, pp. 102-105. Computing, Chapter 5, pp. 153-181, Kluwer Academic Publishers, 1996.

[3] D.B. Johnson and D.A.Maltz, Dynamic source routing in ad hoc wireless networks, in: Mobile Computing, eds. T. Imielinski and H. Korth (Kluwer Academic, 1996) chapter 5, pp. $153-181$.

[4] Juan Carlos Cano and Pietro Manzoni, "Evaluating the energy-consumption reduction in a MANET by dynamically switching-off network interfaces," in Proc. of the 6th IEEE Symposium on Computers and Communications, July 2001.

[5] D.A. Maltz, J. Broch, J. Jetcheva and D.B. Johnson, The effects of on-demand behavior in routing protocols for multihop wireless ad hoc networks, IEEE Journal on Selected Areas in Communications (August 1999).

[6] Y. Xu, J. Heidemann, and D. Estrin. Adaptive EnergyConserving Routing for Multihop Ad Hoc Networks. Technical Report 527, USC/Information Sciences Institute, 2000. 
[7] C.F. Chiasserini, R.R. Rao, A distributed power management policy for wireless ad hoc networks, in: IEEE Wireless Communication and Networking Conference, 2000, pp. 1209-1213.

[8] T. Simunic, H. Vikalo, P. Glynn, G.D. Micheli, "Energy efficient design of portable wireless systems", in: Proceedings of the International Symposium on Low Power Electronics and Design, 2000, pp. 49-54.

[9] Y. Xu, J. Heidemann, D. Estrin, Geography-informed energy conservation for ad hoc routing, in: Proceedings of the International Conf. on Mobile Computing and Networking, 2001, pp. 70-84.

[10] Eun-Sun Jung and Nitin H. Vaidya, "An Energy Efficient MAC Protocol for Wireless LANs", IEEE INFOCOM 2002, New York, U.S.A., June 2002.

[11] Eun-Sun Jung and Nitin Vaidya.,"A Power Saving MAC Protocol for Wireless Networks", Technical Report, July 2002.

[12] E. Shih, P. Bahl, and M. J. Sinclair. Wake on Wireless: An Event Driven Energy Saving Strategy for Battery Operated Devices. In ACM MobiCom 2002, September 2002.

[13] R. Zheng and R. Kravets. On-Demand Power Management for Ad Hoc Networks. In Proc. IEEE INFOCOM, pages 481-491, 2003.

[14] L. Bao and J. J. Garcia-Luna-Aceves, “Topology management in ad hoc networks," in 4th ACM International Symposium on Mobile Ad Hoc Networking and Computing (MobiHoc), June 2003.
[15] Tanomsak Khacharoen, Anan Phonphoem, "A Power Saving Mechanism in Ad Hoc Network with Quality of Service Support", International Conference on Information and Communication Technologies (ICT 2003), Bangkok, Thailand, April 2003, p119-124.

[16] Z. Li and B. Li, "Probabilistic Power Management for Wireless AdHoc Networks," Mobile Networks and Applications, vol. 10, no. 5, pp. 771-782, 2005.

[17] C. Sengul and R. Kravets, "Conserving Energy with OnDemand Topology Management," Proc. Second IEEE Int'l Conf. Mobile Ad Hoc and Sensor Systems (MASS '05), pp. 10-19, 2005

[18] S. Lim, C. Yu, and C. Das, "Rcast: A Randomized Communication Scheme for Improving Energy Efficiency in Mobile Ad Hoc Networks," Proc. 25th IEEE Int'l Conf. Distributed Computing Systems (ICDCS '05), pp. 123-132, 2005.

[19] J. Dorsey and D. Siewiorek, "802.11 Power Management Extensions to Monarch ns," Technical Report CMU-CS-04183, School of Computer Science, Carnegie Mellon Univ., Dec. 2004.

[20] S. Lim, Chansu Yu, and Chita R. Das, "RandomCast: An Energy-Efficient Communication Scheme for Mobile Ad Hoc Networks", IEEE TRANSACTIONS ON MOBILE COMPUTING, VOL. 8, NO. 8, AUGUST 2009.

[21] NS2 manual http://www.isi.edu/nsnam/ns/nsdocumentation.html. 